\title{
Investigation of the shapes of cuts in a plate in contact with a rigid stamp
}

\author{
Akmaral Abdigaliyeva ${ }^{1}$, Venera Bigaliyeva ${ }^{1}$, Sharaina Zholdassova $^{2}$, Daryn Shabdirov ${ }^{1,}$, and Ainur Shagayeva ${ }^{1}$ \\ ${ }^{1}$ Faculty of Information Technology, Atyrau Oil and Gas University, Atyrau, Kazakhstan \\ ${ }^{2}$ Faculty of Engineering, Atyrau Engineering Humanitarian Institute, Atyrau, Kazakhstan
}

\begin{abstract}
A mathematical model of a contact interaction between a plate and rigid stamp is derived taking into account physical and design details. The plate is considered to have a crack, that changes its form. The problem of the contact is evaluated based on the theory of variational inequalities. The shape of the stamp is assumed to be perpendicular to the plate surface and the Poisson's ratio is between 0 and 0.5 . Analytical formulation of the study consists of transformation equation, boundary conditions and integral equation. The result is used in maximization and minimization problems for choosing extremal shape of the vertical break in the plate.
\end{abstract}

\section{Introduction}

Dynamic contact problems of the theory of elasticity and plasticity have a wide range of applications. It is associated with the study of impact and penetration of obstacles, explosive and hydroexplosive stamping and mechanical processing of materials. The high-strength materials are prone to brittle fracture, the presence of microdefects, structural cuts and pointed cavities significantly affects the strength of structures and can lead to their complete or local destruction. Therefore, studies of the stress-strain state near cuts in thin plates are of theoretical and practical interest.

From the recent studies, it is found that for the contact problems with unknown contact area, a variational approach is effective [1-4]. A variational approach to solving contact problems is based on the formulation of the boundary conditions of contact interaction in the form of variational inequalities with one-sided constraints. The expediency of using inequalities in these problems is explained by the fact that the required boundary is included in the variational statement only implicitly, as the boundary between the regions of "active" and "passive" constraints. Thus, no a priori assumptions are required regarding the topology of the unknown boundary determined after solving the problem.

The problem of determining the shape of a crack in a two-dimensional case based on crack sizes, material orthotropy and shell curvature was considered in [5]. With the development of information technologies, numerical methods, implemented through computer programs, have become relevant in contact problems. A huge number of works have been published on numerical modeling and its application in problems of dynamic contact, for example, [6-12].
The properties of the solution to the problem of contact of a plate without cuts with a rigid stamp were studied in [13]. Whereas, authors in [14] investigated the problems of equilibrium of plates with cuts.

In this paper, a plate with a limited area median plane is studied. The plate interacts with a rigid stamp and the set of contact points is unknown. It is assumed that the plate has a vertical break, the shape of which can change. It is required to find the form of cuts that delivers the maximum functionality defined for the solution. For each fixed shape of the cut, the plate deflection is uniquely determined. It is necessary to specify the shape at which the deflection of the plate differs from the predetermined one as much as possible. The cut shapes that are the maximum of this functional will be called extremal. The paper substantiates the fundamental possibility of finding extreme forms of sections.

\section{Mathematical model}

\subsection{Formulation of the problem}

$\Omega \subset R^{2}$ is a bounded domain with a smooth boundary $\partial \Omega,(x, y) \in \Omega$. The section shape is described by an equation $y=\varepsilon \Psi(x), x \in(a, b)$, containing a parameter $\varepsilon$. Suppose that the $z$ axis is directed perpendicular to the plane $x, y$ and $z=\Phi(x, y)$ is the shape of the stamp. The condition for the nonpenetration of the plate and the stamp has the form $w_{\varepsilon}(x, y) \geq \Phi(x, y)$, where $w_{\varepsilon}(x, y)$ is the plate deflection corresponding to the cut shown in Figure 1.

\footnotetext{
* Corresponding author: chdaryn@mail.ru
} 


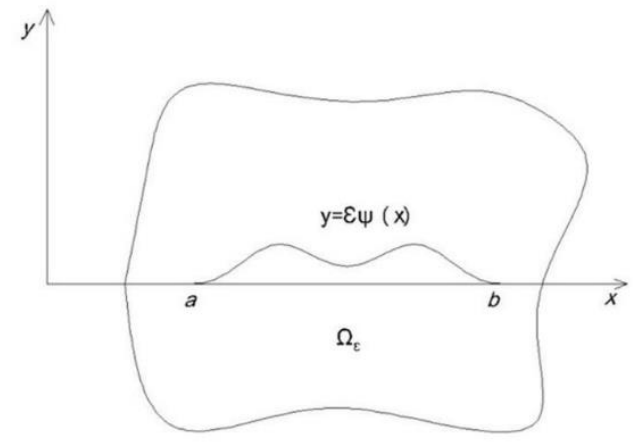

Fig. 1. The plate deflection to the cut.

Figure 2 shows a vertical cross-section of the plate and stamp, where the shape of the stamp is shaded.

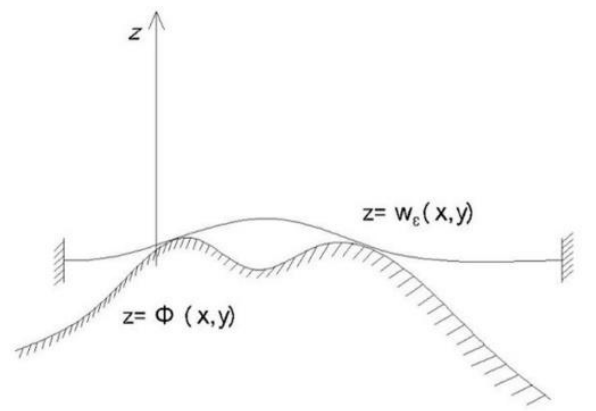

Fig. 2. Vertical cross-section of the plate and stamp.

$H_{0}^{2, \varepsilon}\left(\Omega_{\varepsilon}\right)$ is the space of Sobolev functions having two square-summable generalized derivatives at $\Omega_{\varepsilon}$ and equal to zero together with the first derivatives on $\partial \Omega$. The bilinear form and set of permissible deflections are introduced as following, respectevely:

$$
\begin{gathered}
B_{\varepsilon}\left(D_{u}^{2}, D_{v}^{2}\right)=\int_{\Omega_{\varepsilon}}\left\{u_{x x} v_{x x}+u_{y y} v_{y y}+\right. \\
\left.+\sigma\left(u_{x x} v_{y y}+u_{y y} v_{x x}\right)+2(1-\sigma) u_{x y} v_{x y}\right\} d \Omega_{\varepsilon} \\
K_{\varepsilon}^{\Phi}=\left\{w \in H_{0}^{2, \varepsilon}\left(\Omega_{\varepsilon}\right) \mid w(x, y) \geq \Phi(x, y),(x, y) \in \Omega_{\varepsilon}\right\}
\end{gathered}
$$

where $\sigma$ is Poisson's ratio and $0<\sigma<\frac{1}{2} ; \Phi \in H^{2}(\Omega)$ and $\Phi \mid \partial \Omega<0$. The problem of the contact between the plate and the stamp can be stated as a variational

$$
\min _{w \in K_{\varepsilon}}\left\{\frac{1}{2} B_{\varepsilon}\left(D_{w}^{2}, D_{w}^{2}\right)-(F, W)_{\varepsilon}\right\}
$$

where $F \in L^{2}(\Omega)$ is the specified external load on the plate, and $(F, w)_{\varepsilon}=\int F w d \Omega_{\varepsilon}$. For each fixed $\varepsilon$ problem (1) has a unique solution - plate deflection $w_{\varepsilon}$. This problem is equivalent to the variational inequality.

$$
\begin{gathered}
w_{\varepsilon} \in K_{\varepsilon}^{\Phi}: \\
B_{\varepsilon}\left(D^{2} w_{\varepsilon}, D^{2} \bar{w}_{\varepsilon}-D^{2} w_{\varepsilon}\right) \geq\left(F, \bar{w}_{\varepsilon}-w_{\varepsilon}\right)_{\varepsilon}, \\
\forall \bar{w}_{\varepsilon} \in K_{\varepsilon}^{\Phi} .
\end{gathered}
$$

The boundedness of solutions to problem (2) in the norm $\|\cdot\|_{2, \Omega_{\varepsilon}}$ of space $H_{0}^{2, \varepsilon}\left(\Omega_{\varepsilon}\right)$ can be shown. For this an arbitrary function $\bar{w} \in H_{0}^{2}(\Omega)$, satisfying the inequality $\bar{w} \geq \Phi$ in $\Omega$ is chosen. Then the restriction of this function to $\Omega_{\varepsilon}$ will satisfy the inequality $\bar{w}_{\varepsilon} \geq \Phi$ in $\Omega_{\varepsilon}$, thus $w_{\varepsilon} \in K_{\varepsilon}^{\Phi}$ is valid. By substituting these functions $\left(\bar{w}_{\varepsilon}\right)$ in $(2), B_{\varepsilon}\left(D^{2} w_{\varepsilon}, D^{2} w_{\varepsilon}\right) \leq C$ is obtained, where the constant $C>0$ does not depend on $\varepsilon$. Hence follows the estimate of $w_{\varepsilon}$ :

$$
\left\|w_{\varepsilon}\right\|_{2, \Omega_{\varepsilon}} \leq C
$$

\subsection{Solution for $\varepsilon \rightarrow 0$}

In order to investigate the case $\varepsilon \rightarrow 0$, the the region $\Omega_{\varepsilon}$ is mapped onto a fixed region $\Omega_{o}$. It is required to find the limit of the solution at $\varepsilon \rightarrow 0$ in a fixed region of variation of independent variables. In addition to the discussed parameters, it is assumed that $\Psi \in H_{o}^{\zeta}(a, b)$. The subdomains $\Omega_{1}, \Omega_{2}$ are chosen so that $\bar{\Omega}_{1} \subset$ $\Omega_{2}, \bar{\Omega}_{2} \subset \Omega$ and $\Gamma_{\varepsilon} \subset \Omega_{1}$ for all sufficiently small $\varepsilon$. The function $h$ is such that $h \equiv 1$ on $\Omega_{1}, h \in C_{o}^{\infty}\left(\Omega_{2}\right)$. Consider the transformation of independent variables

$$
\ell=x, y / \sigma y-\varepsilon \Psi h
$$

where the function $\Psi$ continues by zero outside the interval $(a, b)$. For all small values of $\varepsilon$, the Jacobian of this transformation $1-\varepsilon \Psi h_{y}$ is greater than zero. When $u_{\varepsilon}(\tilde{x}, \tilde{y}) \equiv w_{\varepsilon}(x, y), F_{\varepsilon}(\tilde{x}, \tilde{y}) \equiv F(x, y)$, variational inequality (2) in variables $\tilde{x}, \tilde{y}$ takes the form

$$
\begin{aligned}
& B_{o}\left(\lambda_{\varepsilon} D^{2} u_{\varepsilon}, \lambda_{\varepsilon} D^{2} \bar{u}_{\varepsilon}-\lambda_{\varepsilon} D^{2} u_{\varepsilon}\right)+ \\
& +\varepsilon \int_{\Omega_{o}} g\left(\ell, 0 \oint, \rho \varepsilon, D^{\alpha} u_{\varepsilon}, D^{\alpha} \bar{u}_{\varepsilon}\right) \lambda_{\varepsilon}^{2} d \Omega_{o} \geq \\
& \geq\left(\lambda_{\varepsilon} F_{\varepsilon}, \lambda_{\varepsilon} \bar{u}_{\varepsilon}-\lambda_{\varepsilon} u_{\varepsilon}\right)_{o} \forall \bar{u}_{\varepsilon} \in K_{o}^{\Phi_{\varepsilon}}
\end{aligned}
$$

Where $\Phi_{\varepsilon}(\tilde{x}, \tilde{y}) \equiv \Phi(x, y), \lambda_{\varepsilon}=\left(1-\varepsilon \Psi h_{y}\right)^{-\frac{1}{2}}, \quad|\alpha| \leq$ $2 ; \quad K_{o}^{\Phi_{\varepsilon}}=\left\{u \in H_{o}^{2,0}\left(\Omega_{o}\right) \mid u(\tilde{x}, \tilde{y}) \geq\right.$

$$
\left.\Phi_{\varepsilon}(\tilde{x}, \tilde{y}),(\tilde{x}, \tilde{y}) \in \Omega_{o}\right\}
$$

The dependence of a function $g$ on its variables is determined by the nature of the change of variables (4) and the form of inequality (2). The estimate (3) obtained above can be written as follows in the variables $\tilde{x}, \tilde{y}$

$$
\left\|u_{\varepsilon}\right\|_{2, \Omega_{o}} \leq C
$$

with a constant $C$ independent of $\varepsilon,|\varepsilon| \leq \varepsilon_{o}$. Note also that $\Phi_{\varepsilon} \rightarrow \Phi$ uniformly in $\Omega$. This fact (similarly, see [15]) allows to prove that for any element $\bar{u} \in K_{o}^{\Phi}$ there exists a sequence $\bar{u}_{\varepsilon} \in K_{o}^{\Phi_{\varepsilon}}$ such that $\bar{u}_{\varepsilon} \rightarrow \bar{u}$ strongly in $H_{o}^{2,0}\left(\Omega_{o}\right)$.

From the sequence $u_{\varepsilon}$, for any sequence denoted in the same way following works :

$$
u_{\varepsilon} \rightarrow u \text { weakin } H_{o}^{2,0}\left(\Omega_{0}\right)
$$


For an arbitrary fixed element $\bar{u} \in K_{o}^{\Phi}$ on the basis of discribed statemens, a sequence $\bar{u}_{\varepsilon} \in K_{o}^{\Phi_{\varepsilon}}$ that converges strongly to $\bar{u}$ in $H_{o}^{2,0}\left(\Omega_{o}\right)$ is chosen. By taking into account the strong convergence $\lambda_{\varepsilon} \rightarrow 1$ in $L^{2}(\Omega)$, it is possible to pass to the upper limit :

$$
u \in K_{o}^{\Phi}: B_{o}\left(D^{2} u, D^{2} \bar{u}\right) \geq(F, \bar{u}-u)_{o}, \forall \bar{u} \in K_{0}^{\Phi}
$$

This means that the limit function $u$ satisfies the inequality corresponding to $\varepsilon=0$. The result obtained can be formulated as follows: from the sequence of solutions $w_{\varepsilon} \equiv u_{\varepsilon}$ to problem (2), a subsequence can be chosen that satisfies (7), and the limit element $u$ satisfies the variational inequality (8).

\subsection{Maximization and minimization problems}

Using the proved statement, the problem of choosing an extremal shape cut we can be posed. $y=\Psi(x)$ describes the shape of the cut, where $\psi \in \Psi, \Psi \subset$ $H_{o}^{\zeta}(a, b)$ is a given bounded, convex and closed set. For $\psi \in \Psi$ the solution of a problem of the form (2) can be denoted by $w_{\Psi}$. The plate deflection satisfies the relations

$$
\begin{gathered}
w_{\Psi} \in K_{\Psi}^{\Phi}: B_{\Psi}\left(D^{2} w_{\Psi}, D^{2} \bar{w}-D^{2} w_{\Psi}\right) \geq \\
\geq\left(F, \bar{w}-w_{\Psi}\right)_{\Psi}, \forall \bar{w} \in K_{\Psi}^{\Phi}
\end{gathered}
$$

where $K_{\Psi}^{\Phi}=\left\{w \in H_{o}^{2, \Psi}\left(\Omega_{\Psi}\right) \mid w(x, y) \geq\right.$

$$
\left.\Phi(x, y),(x, y) \in \Omega_{\Psi}\right\},
$$

the sign $\Psi$ at the bottom means that the integration is carried out over the area of $\Omega_{\Psi}=\Omega \backslash \Gamma_{\Psi}, \Gamma_{\Psi}$ - the graph of the function $y=\Psi(x)$.

The next task is to formulate the problem of finding a cut shape $y=\Psi(x), \psi \in \Psi$ for the function $w_{o} \in L^{2}(\Omega)$, where the corresponding plate deflection $w_{\Psi}$ differs from $w_{o}$ as much as possible in a given norm. For this the functional is defined as following:

$J(\Psi)=\left\|w_{\Psi}-w_{o}\right\|_{o, \Omega_{\Psi}}$, where $\|\bullet\|_{o, \Omega \Psi}$ is the norm in $L^{2}\left(\Omega_{\psi}\right)$. It is required to solve the maximization problem by showing the cut shape that provides the maximum functionality $J$.

$$
\sup _{\psi \in \Psi} J(\Psi)
$$

$\Psi_{n} \in \Psi$ is a maximizing sequence, so the sequence has the property $J\left(\Psi_{n}\right) \rightarrow \sup _{\psi \in \Psi} J(\Psi)$. The set $\Psi$ is limited, so the sequence $\Psi_{n}$ is limited in $H_{o}^{\zeta}(a, b)$ Without diminishing the generality, consider $\Psi_{n} \rightarrow \Psi$ in $H_{o}^{\zeta}(a, b)$. For each $\Psi n$, the deflection $w_{n}=w_{\Psi_{n}}$ can be found as a solution to the problem (11):

$$
\begin{aligned}
w_{n} & \in K_{\Psi_{n}}^{\Phi}: B_{\Psi_{n}}\left(D^{2} w_{n}, D^{2} w-D^{2} w_{n}\right) \geq \\
& \geq\left(F, \bar{w}-w_{n}\right)_{\Psi_{n}} \forall \bar{w} \in K_{\Psi_{n}}^{\Phi}
\end{aligned}
$$

The domains $\Omega_{1}, \Omega_{2}$ and the function $h$ are chosen with previous criteria, and $\Gamma_{\Psi_{n}} \subset \Omega_{1}$ for all $n$. Assuming that $\Psi, \Psi_{n}$ are continued by zero outside $(a, b)$, consider the transformation of independent variables

$$
\ell / \sigma x, \mathscr{f} \sigma y+\left(\Psi-\Psi_{n}\right) h
$$

Using the embedding theorems, it is assumed that $\left|\Psi_{n x x}-\Psi_{x x}<\frac{1}{n}\right|$ in $(a, b)$. Then transformations (12) can be written in the form $\tilde{x}=x, \tilde{y}=y-\frac{1}{n} \phi_{n} h$, where $\phi_{n}=n\left(\Psi_{n}-\Psi\right)$ is the function constrained in $C^{2}[a, b]$. The Jacobian $1-\frac{1}{n} \phi_{n} h_{y}$ of this transformation is positive for large enough values of $n . u_{n}(\tilde{x}, \tilde{y}) \equiv$ $w_{n}(x, y)$ and inequality (11) in variables $\tilde{x}, \tilde{y}$ has the form

$$
\begin{aligned}
& B_{\Psi}\left(\lambda_{n} D^{2} u_{n}, \lambda_{n} D^{2} \bar{u}-\lambda_{n} D^{2}+\right. \\
+ & \frac{1}{n} \int_{\Omega_{\Psi}} g\left(\begin{array}{l}
\left.\ell, 0 y, 0-\frac{1}{n}, D^{\alpha} u_{n}, D^{\alpha} \bar{u}\right) \\
n
\end{array} \lambda_{n}^{2} d \Omega_{\Psi} \geq\right. \\
\geq & \left.\lambda_{n} F_{n}, \lambda_{n} \bar{u}-\lambda_{n} u_{n}\right)_{\Psi} \forall \bar{u} \in K_{\Psi}^{\Phi_{n}}
\end{aligned}
$$

where $F_{n}(\tilde{x}, \tilde{y}) \equiv F(x, y), \Phi_{n}(\tilde{x}, \tilde{y}) \equiv \Phi(x, y)$,

$$
\lambda_{n}=\left(1-\frac{1}{n} \phi_{n} h_{y}\right)^{-1 / 2}
$$

The estimate of the solution $u_{n}$ in space $H_{o}^{2, \Psi}\left(\Omega_{\Psi}\right)$ has the form $\left\|u_{n}\right\|_{2, \Omega_{\psi}} \leq C$ with a constant $C$ independent of $n$. Choosing a subsequence, if necessary, it is assumed that $\mathrm{u}_{n} \rightarrow \mathrm{u}$ is weak in $H_{0}^{2 \psi}\left(\Omega_{\psi}\right)$ and is strong in $L^{2}\left(\Omega_{\psi}\right)$. In addition, for any element $\bar{u} \in$ $K_{\psi}^{\phi}$ there exists a sequence $\bar{u}_{n} \in K_{\psi}^{\Phi}$ strongly converging in $H_{0}^{2 \psi}\left(\Omega_{\psi}\right)$ to $\bar{u}$. This allows the passage to the limit as $n \rightarrow \infty$ in (13). Namely, following statements are obtained in the limit :

$$
u \in K_{\psi}^{\Phi}, B_{\psi}\left(D^{2} u, D^{2} \bar{u}-D^{2} u\right) \geq(F, \bar{u}-u)_{\psi}, \quad \forall \bar{u} \in K_{\psi}^{\Phi} .
$$

The resulting inequality means that the constructed function $u$ corresponds to the function $\psi$ and the shape of the stamp $z=\Phi$, so $u=u_{\psi}$.

$$
\text { If } w_{\text {on }}(\tilde{x}, \tilde{y}) \equiv w_{0}(x, y) \text {, then }
$$

$$
\begin{aligned}
& \sup _{\bar{\psi} \in \Psi} J(\bar{\psi})=\overline{\lim } J\left(\psi_{n}\right)= \\
& \overline{\lim }\left\|w_{n}-w_{0}\right\|_{0, \Omega_{n}}=\overline{\lim }\left\|\lambda_{n}\left(u_{n}-w_{0 n}\right)\right\|_{0, \Omega_{\psi}}= \\
& \left\|u_{\psi}-w_{0}\right\|_{0, \Omega_{\psi}}=J(\Psi) \leq \sup _{\psi \in \Psi} J(\psi)
\end{aligned}
$$

Therefore, the function $\Psi$ solves the maximization problem (10). It is also possible to raise the question of cuts in which the stress state in the plate is as close as possible to the predetermined one. This leads to a minimization problem of the following form. If $w_{1} \in$ $H^{1}(\Omega)$ is a given function, the functional is $J_{1}(\Psi)=$ $\left\|w_{\Psi}=w_{1}\right\|_{1, \Omega_{\Psi}}$ where $\|\bullet\|_{1, \Omega_{\Psi}}$ is the norm in $H^{1}\left(\Omega_{\Psi}\right)$, 
and $w_{\Psi}$ is the solution of inequality (9) for a given $\Psi$. It is required to find a solution to the problem

$$
\inf _{\psi \in \Psi} J_{1}(\Psi)
$$

It is required to estimate the value of the first derivatives of the solution. It can be shown that there is a function $\psi \in \Psi$ that minimizes the functional $J_{1}$ on the set $\Psi$. There are other problems for which similar statements are valid.

\section{Conclusion}

As a result of this study, the existence of cut forms that give a maximum and minimum to the functional $J(\Psi)$ on the set $\Psi$ is established. The main outcomes can be summarized as follows :

a) The bilinear form and set of permissible deflections are determined by the space of Sobolev function and Poisson's ratio, $0<\sigma<\frac{1}{2}$

b) The problem of the contact between the plate and the stamp is a variational inequality

c)For each fixed parameter $\varepsilon$ problem has a unique solution - plate deflection $w_{\varepsilon}$

d)The solutions of the inequality is boundedness in the norm of space

e) The dependence of a function $g$ on its variables is determined by the nature of the change of variables and the form of inequality

f) for any element $\bar{u} \in K_{o}^{\Phi}$ there exists a sequence $\bar{u}_{\varepsilon} \in K_{o}^{\Phi_{\varepsilon}}$ such that $\bar{u}_{\varepsilon} \rightarrow \bar{u}$ strongly in $H_{o}^{2,0}\left(\Omega_{o}\right)$ and $u_{\varepsilon} \rightarrow u$ weak in $H_{o}^{2,0}\left(\Omega_{0}\right)$

g) for any element $\bar{u} \in K_{\psi}^{\Phi}$ there exists a sequence $\bar{u}_{n} \in K_{\psi}^{\Phi}$ strongly converging in $H_{0}^{2 \psi}\left(\Omega_{\psi}\right)$ to $\bar{u}$

h) the extremal cut shapes are found by the function $\Psi$, which solves the maximization and minimization problems

$$
\sup _{\psi \in \Psi} J(\Psi) \text { and } \inf _{\psi \in \Psi} J_{1}(\Psi) \text {, respectively }
$$

\section{References}

1. Thong V.D. and D.V.Hieu. Weak and strong convergence theorems for variational inequality problems. Numerical Algorithms 78. 1045-1060 (2018)

2. Gibali, A., Reich, S., Zalas, R.: Outer approximation methods for solving variational inequalities in Hilbert space. Optimization 66, 417-437 (2017)

3. Hieu, D.V., Anh, P.K., Muu, L.D.: Modified hybrid projection methods for finding common solutions to variational inequality problems. Comput. Optim. Appl. 66, 75-96

4. Cai G, Gibali A, Iyiola O S, Shehu Y. A new double-projection method for solving variational inequalities in Banach spaces. J Optim Theory Appl, 2018, 178: 219-239

5. N. V. Banichuk. Determination of the shape of curvilinear cracks by the small parameter method. Academy of Sciences of the USSR. 1970. №2. S. 130-137.

6. Jouymandi Z, Moradlou F. Extragradient methods for solving equilibrium problems, variational inequalities, and fixed point problems. Numer Funct Anal Optim, 2017, 38: 1391-1409

7. Liu ZH, Migórski S, Zeng SD. Partial differential variational inequalities involving nonlocal boundary conditions in Banach spaces. J Differ Equ. 2017;263:3989-4006. 10.1016/j.jde.2017.05.010

8. Nguyen TVA, Tran DK. On the differential variational inequalities of parabolic-elliptic type. Math Method Appl Sci. 2017. doi:10.1002/mma.4334

9. Zeng SD, Liu Z, Migórski S. A class of fractional differential hemivariational inequalities with application to contact problem. Z Angew Math Phys. 2018;69:36. pages 23. doi: 10.1007/s00033018-0929-6

10. A. I. Furtsev, "About the Contact of a Thin Obstacle and a Plate Containing a Thin Inclusion," Sibir. Zh. Chist. Prikl. Mat. 17 (4), 94-111 (2017)

11. A. M. Khludnev, "On Modeling Thin Inclusions in Elastic Bodies with a Damage Parameter," Math. Mech. Solids. 2018; doi https://doi.org/10.1177/1081286518796472

12. Giovanardi B, Formaggia L, Scotti A, Zunino P. Unfitted FEM for modelling the interaction of multiple fractures in a poroelastic medium. In: SPA Bordas, E Burman, MG Larson, MA Olshanskii, eds. Geometrically Unfitted Finite Element Methods and Applications. Cham, Switzerland: Springer International Publishing; 2017: 331- 352.

13. Caffarelli L. A, Friedman A. The obstacle problem for the biharmonic operator. Ann scuola norm. super Pisa. 1979, Ser. IV.- vol. VI, no. 1, - P. 151-184.

14. Morozov N.F. Mathematical problems in the theory of cracks. Moscow: Nauka.1984. 255 p.

15. Khludnev A.M. Optimal control of a variational inequality in a contact problem for a plate. Dynamics of a continuous medium. Novosibirsk, 1988. - Issue. 87 .-- S. 122-135.. 